\title{
Characterization of the Gas Dispersion Behavior of Multiple Impeller Stages by Flow Regime Analysis and CFD Simulations
}

\author{
Sören Bernauer ${ }^{1}$, Mathias Schöpf ${ }^{1}$, Philipp Eibl ${ }^{2}$, Christian Witz ${ }^{2}$, Johannes Khinast ${ }^{2}$, and \\ Timo Hardiman ${ }^{1}$ \\ ${ }^{1}$ Sandoz $\mathrm{GmbH}$ \\ ${ }^{2}$ Technische Universität Graz
}

July 6, 2020

\begin{abstract}
Multiple impeller reactors are widely used due to their advanced gas utilization and an increased volumetric mass transfer coefficient. However, with the application of Rushton impellers, gas dispersion efficiency varies between the bottom and the upper impeller levels. The present study analyzes the individual flow regime, power input and gas hold-up in each compartment of a reactor equipped with four Rushton impellers. The results indicate that the pre-dispersion of the air introduced by the bottom impeller plays a key role in a better gas retention efficiency of the upper impellers. In contrast, a flooded bottom impeller adversely affects the gas dispersion of all impellers. A novel analysis of the bubble flow in the dispersed state via a two-phase CFD model reveals that a more homogenous distribution of air bubbles in the upper compartments leads to high compartment gas hold-up values, but fewer bubbles in the vicinity of the impellers. The measured and simulated data of this study indicate that the upper impellers' efficiency mostly depends on the flow regime of and the pre-dispersion by the bottom impeller rather than on the upper impellers' flow regimes. These results contribute to the understanding of essential mixing processes and scaling of aerated bioreactors.
\end{abstract}

\section{Introduction}

Pilot- and large-scale fermentation processes are mostly carried out in bioreactors equipped with multiple impellers. They provide better gas utilization with higher gas phase residence times, an increased gas holdup, and thus, a higher volumetric mass transfer coefficient (Gogate, Beenackers, \& Pandit, 2000). Despite the variety of new impeller models, the most common impeller for microbial fermentation and for educational purposes is still the Rushton turbine. The bottom impeller has been shown to behave differently from the upper impellers regarding flow regime and the resulting oxygen mass transfer coefficient and power input. For instance, several studies showed that the gas hold-up in case of a multiple impeller reactor is higher in the upper impeller compartments than in the bottom impeller compartment (Gogate et al., 2000; Linek, Moucha, \& Sinkule, 1996; Nocentini, Magelli, Pasquali, \& Fajner, 1988). Gogate et al. (2000) assumed that gas hold-up in the second impeller compartment is about $30-40 \%$ higher than in the bottom compartment and Linek et al. (1996) determined an increase of about $15 \%$ on average for the upper compartments, paired with a higher total oxygen mass transfer coefficient of about $40 \%$ in a four-level reactor.

At the same time, numerous studies showed that the bottom impeller exhibits a more pronounced power drop, when aerated than the upper impellers (Hari-Prajitno et al., 1998; Linek et al., 1996; Middleton \& Smith, 2004; Warmoeskerken \& Smith, 1988). Linek et al. (1996) measured an about $50 \%$ higher power draw by the upper impellers. Nienow and Lilly (1979) assumed that the reason for this behavior is that 
the number of air bubbles passing the upper impellers must be significantly lower than those at the bottom impeller.

When aerated, Rushton impellers form cavities behind the impeller blades. Extensive studies (Bombac, Zun, Filipic, \& Zumer, 1997; Bruijn, Vantriet, \& Smith, 1974; Nienow, Warmoeskerken, Smith, \& Konno, 1985) analyzed the different forms and transitions of the cavities in relation to impeller speed and aeration rate. In the so-called vortex cavity (VC) regime, which appears at low aeration rates and high impeller speeds, vortices behind the impeller blades disperse the air. In the loaded state at higher aeration rates, the gas accumulates in the low-pressure zone behind each impeller and forms large cavities (LC). With a further increase in aeration rate, the large cavities grow until reaching the next impeller blade resulting in the ragged cavity regime (RC), or also called "flooding". Typically, flooding occurs earlier at lower impeller speeds. The transitions between these flow regimes have been studied comprehensively for various operating conditions and reactor geometries for reactors with one impeller (Bombac et al., 1997; Lee \& Dudukovic, 2014; Lu \& Ju, 1989; Nienow, Wisdom, \& Middleton, 1977; Warmoeskerken \& Smith, 1985). Only few studies investigated the flow regimes in multiple impeller reactors (Abrardi, Rovero, Baldi, Sicardi, \& Conti, 1990; Bombac \& Zun, 2006; Smith, Warmoeskerken, \& Zeef, 1987; Warmoeskerken \& Smith, 1988). These studies examined only a narrow operational range and the flow regimes impact has been studied only with regard to power input.

An increasing number of publications analyze the gas-liquid flow in bioreactors by means of computational fluid dynamics (CFD), focusing for example on flow patterns of the gas and liquid phase (Bakker \& Oshinowo, 2004; Guan, Li, Yang, \& Liu, 2019; Khopkar, Rammohan, Ranade, \& Dudukovic, 2005; Khopkar \& Tanguy, 2008; Scargiali, D'Orazio, Grisafi, \& Brucato, 2007; Wang et al., 2014). Khopkar and Tanguy (2008) investigated the complex pattern in a dual impeller configuration and simulated gas hold-up and the flow pattern of a VC and LC regime. Furthermore, they were able to show a decreasing liquid pumping efficiency of the bottom impeller. However, none of these studies investigated the mechanisms leading to differences between the impeller levels, which were described in the above-mentioned contributions.

The existing data on the flow regimes in multiple impeller reactors and their impact on the gas dispersion behavior is incomplete. Therefore, in this study, we analyze the power input and gas hold-up of each impeller stage and the corresponding flow regime in a four-level reactor and over a wide, industrially relevant operational range. The second objective of this paper is to study mechanisms leading to the differences between the reactor compartments. By means of a two-phase CFD simulation, it is for the first time possible to analyze the bubble flow in each compartment of a pilot-scale $\left(0.15 \mathrm{~m}^{3}\right)$ bioreactor applying high gas hold-up and turbulent conditions, to determine the effective (local) aeration rate and local gas density at each impeller.

\section{Materials and Methods}

\section{Fermenter}

Experiments were performed in a pilot scale Plexiglas reactor with an inner diameter $(\mathrm{T})$ of $0.44 \mathrm{~m}$ and a working volume of $0.168 \mathrm{~m}^{3}$. The geometrical details of the vessel are displayed in Figure 1. Airflow was introduced through a ring sparger with 36 holes, each diameter of $2 \mathrm{~mm}$, and controlled by a mass flow controller (EL-Flow Select F-203AV, Bronkhorst AG, Netherlands). A $0.065 \mathrm{~mol} / \mathrm{L} \mathrm{Na}_{2} \mathrm{SO}_{4}$ solution was used as model fluid to simulate the non-coalescent behavior present in most fermentation media (Prince \& Blanch, 1990).

\section{Experimental methods}

The flow regimes were determined by means of the conductivity method described by Bombac et al. (1997). In order to detect the gas-filled cavities behind the impeller blades, and thus, the respective flow regime, a 
self-made 2-pole conductivity probe with small tip size was applied (Figure 1). The probe was mounted on an in height-adjustable holder, which was moved to the respective impeller level for measurements (Witz, Treffer, Hardiman, \& Khinast, 2016). The cavity size and type behind each blade were identified by a drop in the voltage pulse response of the probe. For this, the raw signal was further analyzed by discrete Fourier transformation using PicoScope software (Version 6.9.14.16, Pico Technology Ltd., United Kingdom).

In a first set of experiments, the flow regimes were recorded covering aeration rates between 2 and $18 \mathrm{~m}^{3} / \mathrm{h}$ and gas superficial velocities $\left(u_{g}\right)$ between 0.004 and $0.033 \mathrm{~m} / \mathrm{s}$ and impeller speeds from 100 to $440 \mathrm{~min}^{-1}$. In a second set of experiments, each impellers transition lines from VC to LC and from LC to RC were identified. For this, the impeller speed was set to a constant value and the gas flow rate was increased in small steps of $0.1 \mathrm{~m}^{3} / \mathrm{h}$ until a change of the flow regime was recognized. This procedure was repeated at other impeller speeds to be able to draw the transition line. For further analysis of the flow regimes, the transition lines were plotted as a flow map (Warmoeskerken \& Smith, 1985), which employs in the dimensionless Froude $(\mathrm{Fr})$ and Flow number $(\mathrm{Fl})$ and allows a coherent classification of the flow regimes.

The gas hold-up and power measurements were conducted in separate experiments with the conductivity measurement setup removed to avoid an influence on the measurements. The gas hold-up and power input were determined for vessel filling levels covering the impeller levels 1, 1 and 2, 1 to 3 and 1 to 4 (Figure 1). The calculation of the difference between values of the reactor filled with $n$ levels and $n$-1levels yielded the value of level $n$.

The gas hold-up was therefore determined by calculation of the difference of the measured total filling level for aerated conditions for $n$ impeller levels, $H_{t,(n)}$, and for the lower $n-1$ levels, $H_{t,(n-1)}$, as described by Fujasova, Linek, and Moucha (2007) for $n=1,2,3,4$ and the relation

\begin{tabular}{l}
$\varepsilon_{n}=\frac{\left(H_{t,(n)}-H_{t,(n-1)}\right)-\left(H_{l} / 4\right)}{\left(H_{t,(n)}-H_{t,(n-1)}\right)}$ \\
\hline
\end{tabular}

where $H_{l} / 4$ is the filling level for unaerated conditions of each compartment and is equal to $0.625 \mathrm{~T}$. A possible effect of surface aeration is minimized as it is subtracted, except of the bottom impeller where this influence can be assumed to be negligible under aerated conditions (Chapman, Nienow, \& Middleton, 1980).

Power consumption was determined by measurement of the torque $M$ applying a strain gauge with telemetry technique (Trachsler Electronics GmbH, Switzerland) mounted above the top impeller. Thus, $M$ and the resulting represents the total power consumption of all immersed impellers. The torque measurements were conducted in parallel to the gas hold-up measurements leading to the respective power input $P$ of compartment 1-4, 1-3, 1-2 and the bottom compartment. Similar to the gas hold-up determination, each impeller stages' power draw was calculated applying, $P_{n}-P_{(n-1)}$. Below a Fr number of 0.352 the low torsion yielded in high measuring uncertainty of the strain gauge signal and measurements were therefore not considered in this study.

\section{CFD Simulations}

CFD Simulations were conducted applying the Euler-Lagrange approach of the two-phase flow as described in Witz et al. (2016) and Eibl, Rustige, Witz, and Khinast (2020). The Lattice Boltzmann method (LBM) was used for simulation of the liquid flow field and the bubble movement was captured via a Lagrangian approach by solving the Newton's equation of motion with the forces fluid stresses, gravity, drag, lift and added mass forces acting on the bubbles.

The modified half-way bounce back method (Ladd, 1994) was applied for the static boundaries in the LBM, like the reactor walls. The moving boundaries, e.g. the Rushton stirrers, were represented by a wet node boundary approach by Lallemand and Luo (2003). The top surface of the simulation domain was modelled as a free surface using a free slip boundary condition. 
Bubbles with similar properties were grouped together, forming parcels to reduce the memory consumption and hence enabling the simulation of the large number of bubbles inside the reactor. Turbulent eddies in a size range similar to the bubble diameter were assumed to be the main cause for bubble breakup. The daughter bubble size distribution was determined using the model of Luo and Svendsen (1996). The fluid field and the bubbles are two-way coupled, i.e. the fluid experiences feedback forces generated by the bubbles. The displacement of the fluid by the bubbles was not considered.

All dimensions of the simulated reactor are similar to the pilot scale reactor to allow a direct comparison (Figure 1). Two test cases were simulated, the first with an aeration rate $\mathrm{Q}$ of $6 \mathrm{~m}^{3} / \mathrm{h}$ and a superficial gas velocity $\mathrm{u}_{\mathrm{g}}$ of $0.011 \mathrm{~m} / \mathrm{s}$ at an impeller speed of $440 \mathrm{~min}^{-1}(\mathrm{Fr}=0.87, \mathrm{Fl}=0.06)$. The second test case was simulated with $4 \mathrm{~m}^{3} / \mathrm{h}$ aeration and $\mathrm{a} \mathrm{u}_{\mathrm{g}}$ of $0.007 \mathrm{~m} / \mathrm{s}$ at $280 \mathrm{~min}^{-1}(\mathrm{Fr}=0.35, \mathrm{Fl}=0.06)$ impeller speed. The gas hold-up was calculated by

$$
\overline{\varepsilon_{\text {sim }}=\frac{\sum_{i=1}^{n}\left(V_{\text {Bubble }, i}\right)}{V_{\text {Compartment }}}} \quad 2
$$

with the volume of each bubble $V_{\text {Bubble, } i}$ and the volume of one impeller compartment $V_{\text {Compartment }}$ (Figure 1). To distinguish between the ratio of the actual gas passing the impeller to the gas circulating in the compartment and bypassing the impeller, the gas hold-up in the volume of each impeller was calculated. Eq.2 was applied replacing $V_{\text {Compartment }}$ by $V_{\text {Impeller }}$, the volume of the cylinder around each impeller, with the dimensions of the impeller diameter and the impeller blades height (Figure 1). To examine the local gas load of each impeller, the effective aeration rate was introduced. This rate is equal to the radial gas flow leaving the impeller region as dispersed gas. The flow of gas dispersed by each impeller was estimated by multiplying the velocities of the bubbles in the lateral surface of the impellers cylinder with the void fraction of the respective cells. Thus, this key figure reflects the axial aeration at the impeller by the gas bubbles recirculating from top and below of the impeller, and the radial gas dispersion process.

\section{Results}

\section{Flow Regimes}

A flow regime analysis is shown in Figure 2 (left column), where the Fr number is plotted as a function of the Fl number. Measurements at 81 operating points were performed for each impeller level. The analysis reveals that the flow regimes $\mathrm{VC}, \mathrm{LC}$ and RC occurred at all impeller levels, depending on the operating parameters. The position of the transition lines was further analyzed and is displayed in Figure 3 (left). Only at high Fr numbers $(>0.72)$, all impellers exhibit the transition from the vortex to the loaded cavity regime at similar stirring and aeration conditions. For the bottom impeller, the vortex-loaded-transition (cavity line) can be expected to occur at the same Fl number, independent of the Fr number as gas is applied without pre-dispersion to the bottom impeller and the gas load becomes too high. The cavity line moves towards higher Flow numbers, with the increase of fermenter height and number of impellers. This study demonstrates for the first time that this shift of the transition becomes stronger with each impeller level and is most pronounced at the lowest Fr number of 0.18, where the transition moves from a Fl number of 0.03 for the first, to 0.08 for the second, 0.09 for the third and 0.11 for the fourth impeller. This significant difference between the cavity lines of the bottom impeller and the three upper impellers is of particular interest for understanding the dispersion behavior of the whole bioreactor. A straightforward explanation might be that in the non-coalescing media applied in the present study, each impeller stage disperses the rising bubbles more effectively and distributes the gas more homogeneously. Thus, smaller bubbles are observed along the reactor height. This hypothesis helps explaining the individual shift of the vortex-loaded-transition at each impeller level.

When the stirrer speed is high, small bubbles are strongly pumped and accumulate in the recirculation 
flow. Therefore, the upper impellers become loaded already at lower Fl numbers, and, the cavity line of the bottom and the upper impellers approach each other and intersect at a Fl number of 0.03 (Figure 3, left). The observation of an individual and more pronounced shift for the upper impellers is contrary to the results of Smith et al. (1987). These authors used water in a three level reactor for their experiments, which leads to coalescence of the rising air bubbles and could explain why their cavity lines of the upper two impellers were similar to each other.

Despite the experimental differences in their study, the results can be explained in a similar way. These authors (Smith et al., 1987) assumed that the gas in the upper compartments is more uniformly distributed, leading to a lower gas density at the impeller, and thus to a shift in the vortex-loaded-transition. The increase of rotation rate and Fr number, leads to an increased circulation and a concentration of the gas stream arriving at the impeller, and thus to the lack of the shift for high Fr numbers. However, for the non-coalescent medium applied in the present study, the observed shift is individual and more pronounced for the upper impellers (Figure 3) since each compartment contributes to the dispersion of gas bubbles and the impellers of higher levels become less loaded.

A shift towards higher Fl numbers also applies for the transition from the loaded to the flooded flow regime (flooding line) which moves from a Fl number of 0.13 for the bottom impeller, to 0.22 for the second, 0.31 for the third and 0.34 for the fourth impeller at a similar Fr number of 0.26 (Figure 2 and 3). Our analysis of the flooding line at each impeller level extends the results of Bombac and Zun (2006) by using a fourth impeller level. As can be seen, there is a further shift of the flooding line to higher Fl numbers with an increase in impeller level. At the flooded state of the bottom impeller, large bubbles are visible, not being dispersed and rising through the reactor (visible inspection, data not shown). Thus, not all air can be dispersed by the second and following impellers even though they are still in the loaded regime (Figure 2). This phenomenon was already observed by Nocentini et al. (1988) for a lab scale reactor filled with tap and distilled water.

In contrast, the upper impellers still showed a radial gas flow despite the impeller's probe indicated a flooded impeller (visible inspection, data not shown). This raises the question if the upper impellers actually reached a flooded state comparable to the bottom impeller flooding. Detached small bubbles that are formed from large, buoyancy driven bubbles may lead to the observed minor circulation flow in the radial direction.

In conclusion, the upper Rushton turbines of the multistage aerated bioreactor exhibit a shift in the cavity and flooding lines towards higher Fl numbers. While an equal shift of vortex-cavity-transition can be expected for all upper impeller stages in coalescent (water) medium according to Smith et al. (1987), the gas pre-dispersion by the lower impellers appears to cumulatively shift this transition for the upper impellers in the non-coalescent (salt) medium applied in the present study. This individual shift is demonstrated for the first time and its explanation will be critically analyzed in the below CFD simulation study.

\section{Power Input}

In Figure 2 (middle column), the relative power input by each of the impellers (with respect to the unaerated case $\mathrm{P}_{0}$ ) is shown as a function of $u_{g}$. Without aeration (data not shown), every impeller stage draws the same power. Under aerated conditions, the bottom impeller shows a well-known distinct decrease of the relative power input already at low aeration rates. The power decrease is most pronounced at the occurrence of cavities around the transition to the loading regime (square shape in Figure 2). Once every impeller blade has a cavity attached and the cavities grow with an increase in aeration rate (Fl number [?] 0.07), the power decrease is less pronounced.

In contrast, the upper impellers show a moderate decrease of the relative power input. This behavior is most pronounced at higher impeller levels $(3 \& 4)$, indicating that the cavity formation of the upper impellers is spread over a larger range of aeration rates. This moderate relative power decrease mainly occurs in the LC regime, but is present in all studied flow regimes. This makes evident that the drop in the power draw of the upper impellers may not be attributed to specific flow regimes as previously postulated for example by Smith et al. (1987). Our approach of studying the individual power draw of each impeller stage together 
with the respective flow regime enables this new view on the effect of aeration on power input (Figure 2). Again, the differences between the bottom and the upper impellers may be due to the occurrence of less air bubbles close to the upper impellers, leading to a higher density of the gas-liquid dispersion around the impeller, which results in higher power inputs. The below CFD analysis will embrace this hypothesis in more detail.

\section{Gas Hold-up}

In Figure 2, right column, the gas hold-up is shown as a function of $u_{g}$ for each impeller level and for all operating conditions. The upper impellers exhibit a higher gas hold-up than the bottom impeller as expected (Figure 2, right column). Interestingly, the generally desired positive effect on gas hold-up of increasing the impeller speed (Fr) is stronger for the upper impellers compared to the bottom level stirrer. The increase of aeration rate (i.e. $u_{g}$ ) has a positive impact on the gas hold-up and this is individual for each impeller. Both the bottom and second impeller level exhibit only a minor dependence on aeration and thus show a low aeration efficiency. This demonstrates that these impellers are not capable of dispersing the supplied air efficiently, and the surplus air is transferred to the next compartment. A reason for this may be that gas bubbles in the lower two compartments are larger, more buoyancy driven and therefore are not following the recirculation flow to the same extent as the smaller bubbles of the upper compartments. The upper two impellers show a very good aeration efficiency that is illustrated by the steep slope of gas hold-up when increasing aeration rate (Figure 2, right column).

Moreover, the upper impellers and predominantly the fourth level show an interesting behavior when the bottom impeller is flooded: The aeration efficiency is lower when the bottom impeller is in the flooded state. In particular on the fourth level, the gas hold-up stays almost constant with increase in aeration rate (Figure 2, right column, blue lines) and demonstrates that a substantial part of the introduced airflow does not contribute to the gas hold-up. This implies that large bubbles rise to the top of the reactor in case of a flooded bottom impeller, and lead to a low dispersion efficiency for the upper impeller levels.

The observations of the gas hold-up distribution are in contrast to other publications (Linek et al., 1996; Nocentini, Fajner, Pasquali, \& Magelli, 1993), which assumed a similar gas hold-up for the upper impeller levels. Our study thus draws a novel picture of a strong dependency of the upper impellers' gas dispersion performance on the flow regime of the bottom impeller.

Figure 3 (right) summarizes the higher gas hold-up within the upper impeller compartments (as detailed in Figure 2). Besides the gas hold-up, the relative power input also increases with the impeller level. This is an interesting finding as high gas hold-up is usually associated with low power input. Although this is true for the individual stirrer levels, it is not between levels (Figure 3). It may be again concluded that the upper compartments profit from the aforementioned pre-dispersed air rising from the lower compartments, and the resulting homogenous dispersion may correspond with a lower local gas hold-up in the immediate vicinity of the upper impellers. Thus, the individual local gas hold-up at the impeller discs might explain the expected power drop for each impeller, when the compartment gas hold-up increases, and, the better power input for upper impellers compared with lower impellers. Figure 3 (right) illustrates this behavior for a Fr number of 0.46. The qualitatively described behavior was observed over the complete experimental area between $\mathrm{Fr}$ number of 0.28 and 0.87 . The following CFD simulation supports these findings and enables studying the complex mechanism leading to this dispersion behavior.

\section{Computational Analysis}

The experimental results of each impeller's flow regime, power input and gas hold-up show drastic differences between the impeller levels. They further indicate that the local gas bubble distribution within each compartment changes from the bottom to the top compartment. Therefore, we analyzed the local bubble distribution and movement in the individual impeller compartments by means of a two-phase CFD simulation. Furthermore, the estimation of the local gas hold-up throughout the reactor based on the number of 
bubbles, their size and volume was performed. Such information, to the best of our knowledge, has not yet been applied in this detail before for studying gas dispersion in pilot-scale bioreactors.

For comparison, two simulations were conducted, one in the $\mathrm{VC}$ and one in the $\mathrm{LC}$ flow regime of the bottom impeller. The simulated total gas hold-up was $15 \%$ in test case 2 (LC), and $5 \%$ in test case 1 (VC). The simulation exhibits a reasonable correlation with measured gas hold-up values (with a relative standard error of $14 \%$ for test case 1 and $19 \%$ for test case 2). The detailed analysis of the bubble properties and distribution for each impeller level and for both test cases is shown in Figure 4. The number of bubbles,

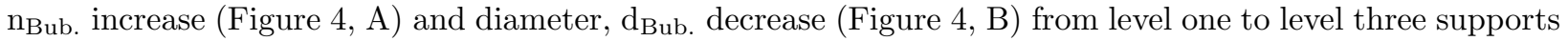
the aforementioned assumption of a better gas dispersion with increasing level. Along with the increase in bubble velocity $\mathrm{v}_{\text {Bub. }}$ (Figure $4, \mathrm{C}$ ), these results substantially support our aforementioned hypothesis for the shifting cavity lines for each additional impeller stage. In test case 1, a decrease in bubble number and an increase in diameter can be seen from level three to level four (Figure $4 \mathrm{~A}$ and B), which may be due to gas recirculation from the level above and leading to a lower amount of bubbles in the fourth compartment. This could not be verified in the experimental set-up. However, in the experiments the gas hold-up of each compartment was derived by a differential approach. The gas hold-up for each level was determined without the presence of the respective next compartment on top of the one studied. In test case 2, this phenomenon cannot be observed in the computations, which can be explained by less recirculation due to larger and thus more buoyancy-driven gas bubbles. The experimentally observed increasing gas hold-up from impeller one to four was also observed in the simulations (Figure $4 \mathrm{~F}$ ). The difference in the bubble size between the impeller levels of test case 1 is only minor and may be explained by reaching a stable droplet size (Figure 4 B).

A comparison of the local gas hold-up, $\varepsilon_{\text {Imp. }}$, in the disk volume of (Figure $4 \mathrm{D}$ ) and of the local gas pumping capacity, QImp., through each impeller (Figure $4 \mathrm{E}$ ) reveal a new picture on the dispersion behavior of the multistage impeller bioreactor. From the first to the second level a significant drop in these key figures can be observed, which implies that the upper impellers are exposed to less gas (i.e., the gas load is lower compared with the bottom impeller). A large fraction of recirculating gas bubbles in the upper compartment seem to bypass the impeller region. This might explain why the local gas hold-up at the impeller is low although the compartment gas hold-up, $\varepsilon_{\text {Comp., }}$ is high (Figure $4 \mathrm{~F}$ ). The higher velocity of bubbles (Figure $4 \mathrm{C}$ ) within the upper impeller levels results in a better gas recirculation and explains the increase in gas hold-up. Test case 1 shows an increase of local gas hold-up and local aeration rate (gas load) from the second to the fourth impeller. This is accompanied by an increase in bubble velocity.

The results of a low local gas hold-up in the impeller region, as well as the significant lower local aeration rate (gas loading) for the upper impellers, agree to our experimental results (Figure 2 and Figure 3) and the assumption of a less pronounced power decrease due to a higher gas-liquid density paired with a higher compartment gas hold-up.

The simulation approach also allows a detailed analysis of the impeller cavities by displaying the simulated gas fraction isosurface (Khopkar \& Tanguy, 2008).

Figure 5 (left) shows the bottom view of each impeller level with the isosurface of a $15 \%$ void fraction (corresponding to the gas hold-up of $>15 \%$ ). Test case 1 with a high Fr number shows large cavities at all impeller levels. The bubbles velocity increases from second to fourth level. Test case 2 with low Fr number shows large cavities at the bottom impeller and vortex cavities for upper impellers. The velocity is lower compared to test case 1 . These results are remarkable as they correspond to the experimentally determined complex flow regimes for these conditions and reinforce the simulations validity. It must be noted that this approximative approach of choosing $15 \%$ void fraction for illustration enables directly comparing gas accumulation for both test cases, although gas cavities exhibit a void fraction of $100 \%$.

The estimated local aeration rates of each impeller level (Figure $4 \mathrm{E}$ ) revealed large differences between the bottom and the upper impellers. Following these results, it is to question if the flow rate introduced by the sparger and the resulting $\mathrm{Fl}$ number is the correct quantity to describe the upper impellers flow regimes. 
The local aeration rates allow the calculation of each impellers actual Fl number and an adjustment of the positions on the flow map. This is illustrated for the two test cases in

Figure 5 (right), where a shift of the adjusted Fl numbers (colored symbols) towards lower values compared to the Fl number calculated for the gas inlet aeration rate (black symbols). This shift is more pronounced for the test case 2 with low impeller speed and shows that the differences of aeration between the upper and the bottom impeller is larger at low Fr numbers. These results support substantially the above experimental flow regime analysis (Figure 2): The shift of the cavity lines is larger at small Fr numbers and the cavity lines of all impeller levels intersect at high Fr numbers. We postulate that the observed shifts in the flow regime maps can be explained by the differences in the effective gas flow resulting from pre-dispersed gas from the lower level. Moreover, at higher stirrer speed, the recirculation is stronger and more gas bubbles are drawn into this flow. Consequently, for the higher local gas hold-up that is observed at the upper impeller levels even more gas bubbles follow this flow. This might lead to the observed intersection of cavity lines at all impeller levels for high Fr numbers.

\section{Conclusions}

The current work presents new insights into stirring and aeration of a pilot-scale multistage Rushton impeller bioreactor. The complementary experimental and simulation analyses indicate that the pre-dispersion of air by the bottom impeller leads to a significantly reduced local gas load at the upper impellers, and thus, to the superior gas utilization efficiency and high power input of the multiple impeller stages. However, the benefits directly depend on the bottom impeller flow regime.

This conclusion is based on several new experimental findings:

- An individual, cumulative shift in the formation of cavities and flooding at the upper impellers towards higher Fl numbers

- A moderate decrease of the relative power input at the upper impellers for elevated aeration rates

- A superior gas utilization efficiency of the upper impellers, i.e. sensitivity of the gas hold-up with respect to stirring and aeration

- An enhanced power input with each impeller level, although the gas hold-up increases

- An individual sensitivity of gas hold-up (and power input) in the upper bioreactor compartments with respect to stirring and aeration, dependent on the bottom stirrer flow regime, and, independent of the local flow regime of the upper impellers

The novel two-phase CFD simulation results support the above conclusion and are consistent with the experimental data:

- The higher gas hold-up in the upper compartments is due to the larger number and smaller size of recirculating gas bubbles

- A large fraction of gas bubbles in the upper compartments is recirculating without moving through the impeller disc region

- The newly introduced local aeration rate reflects this behavior and indicates lower local Fl numbers for the upper impellers. This corresponds to the experimentally demonstrated shift of the flow regime transition towards higher Fl numbers in terms of the overall aeration rate.

The work provides novel aspects towards at mechanistic understanding of the complex gas dispersion and distribution behavior of multiple Rushton impeller bioreactors, particularly of the individual contribution by each impeller stage. Moreover, this knowledge will open up new opportunities for optimizing their design and operating conditions of bioreactors. 


\section{Nomenclature}

$b$ impeller blade length $[\mathrm{m}]$

Bub. bubble

$C$ reactor bottom clearance $[\mathrm{m}]$

CFD computational fluid dynamics

Comp. Compartment

$d$ bubble diameter [mm]

$D$ impeller diameter $[\mathrm{m}]$

Fl Flow number $\mathrm{Fl}=\frac{Q}{N * D^{3}} \quad[-]$

Fr Froude number Fr $=\frac{N^{2} * D}{g}[-]$

$H_{t}$ total aerated liquid height [m]

$H_{l}$ unaerated liquid height $[\mathrm{m}]$

Imp. Impeller

$L$ spacing between impellers $[\mathrm{m}]$

$L B N$ Lattice Boltzman method

LC large cavity regime

$M$ torque [Nm]

$N$ impeller frequency $\left[\mathrm{s}^{-1}\right]$

$P$ power input $[\mathrm{W}]$

$P_{g}$ specific power dissipated by impeller under gassed condition [W]

$P_{0}$ specific power dissipated by impeller under ungassed condition [W]

$\mathrm{RC}$ ragged cavities regime

$S$ sparger height [m]

$T$ tank diameter $[\mathrm{m}]$

$u_{g}$ gas superficial velocity $\left[\mathrm{m} \mathrm{s}^{-1}\right]$

$Q$ aeration rate $\left[\mathrm{m}^{3} \mathrm{~h}^{-1}\right]$

$v$ bubble velocity $\left[\mathrm{m} \mathrm{s}^{-1}\right]$

$V$ volume $\left[\mathrm{m}^{3}\right]$

$\mathrm{VC}$ vortex cavities regime

$w$ impeller blade height $[\mathrm{m}]$

Greek letters

$\varepsilon$ gas hold-up [-] 


\section{Literature}

Abrardi, V., Rovero, G., Baldi, G., Sicardi, S., \& Conti, R. (1990). Hydrodynamics of a Gas-Liquid Reactor Stirred with a Multi-Impeller System. Chemical Engineering Research 83 Design, 68 (6), 516-522.

Bakker, A., \& Oshinowo, L. M. (2004). Modelling of turbulence in stirred vessels using large eddy simulation. Chemical Engineering Research $\&$ Design, 82 (A9), 1169-1178. doi:10.1205/cerd.82.9.1169.44153

Bombac, A., \& Zun, I. (2006). Individual impeller flooding in aerated vessel stirred by multiple-Rushton impellers. Chemical Engineering Journal, 116 (2), 85-95. doi:10.1016/j.cej.2005.10.009

Bombac, A., Zun, I., Filipic, B., \& Zumer, M. (1997). Gas-filled cavity structures and local void fraction distribution in aerated stirred vessel. AICHE Journal, 43 (11), 2921-2931. doi:10.1002/aic.690431105

Bruijn, W., Vantriet, K., \& Smith, J. M. (1974). Power-Consumption with Aerated Rushton Turbines. Transactions of the Institution of Chemical Engineers, 52 (1), 88-104.

Chapman, C. M., Nienow, A. W., \& Middleton, J. C. (1980). Surface Aeration in a Small, Agitated, and Sparged Vessel. Biotechnology and Bioengineering, 22 (5), 981-993. doi:10.1002/bit.260220507

Eibl, P., Rustige, S., Witz, C., \& Khinast, J. (2020). LBM for two-phase (bio-)reactors Advances in Chemical Engineering: Academic Press.

Fujasova, M., Linek, V., \& Moucha, T. (2007). Mass transfer correlations for multiple-impeller gas-liquid contactors. Analysis of the effect of axial dispersion in gas and liquid phases on "local" $\mathrm{k}(\mathrm{L})$ a values measured by the dynamic pressure method in individual stages of the vessel. Chemical Engineering Science, 62 (6), 1650-1669. doi:10.1016/j.ces.2006.12.003

Gogate, P. R., Beenackers, A. A., \& Pandit, A. B. (2000). Multiple-impeller systems with a special emphasis on bioreactors: a critical review. Biochemical Engineering Journal, 6 (2), 109-144. doi:10.1016/s1369$703 x(00) 00081-4$

Guan, X., Li, X., Yang, N., \& Liu, M. (2019). CFD simulation of gas-liquid flow in stirred tanks: Effect of drag models. Chemical Engineering Journal . doi:10.1016/j.cej.2019.04.134

Hari-Prajitno, D., Mishra, V. P., Takenaka, K., Bujalski, W., Nienow, A. W., \& McKemmie, J. (1998). Gasliquid mixing studies with multiple up- and down-pumping hydrofoil impellers: Power characteristics and mixing time. Canadian Journal of Chemical Engineering, 76 (6), 1056-1068. doi:10.1002/cjce.5450760612

Khopkar, A. R., Rammohan, A. R., Ranade, V. V., \& Dudukovic, M. P. (2005). Gas-liquid flow generated by a Rushton turbine in stirred vessel: CARPT/CT measurements and CFD simulations. Chemical Engineering Science, 60 (8-9), 2215-2229. doi:10.1016/j.ces.2004.11.044

Khopkar, A. R., \& Tanguy, P. A. (2008). CFD simulation of gas-liquid flows in stirred vessel equipped with dual Rushton turbines: influence of parallel, merging and diverging flow configurations. Chemical Engineering Science, 63 (14), 3810-3820. doi:10.1016/j.ces.2008.04.039

Ladd, A. J. C. (1994). Numerical Simulations of Particulate Suspensions Via a Discretized Boltzmann-Equation .1. Theoretical Foundation.Journal of Fluid Mechanics, 271 , 285-309. doi:10.1017/S0022112094001771

Lallemand, P., \& Luo, L. S. (2003). Lattice Boltzmann method for moving boundaries. Journal of Computational Physics, 184 (2), 406-421. doi:10.1016/S0021-9991(02)00022-0

Lee, B. W., \& Dudukovic, M. P. (2014). Determination of flow regime and gas holdup in gas-liquid stirred tanks. Chemical Engineering Science, 109 , 264-275. doi:10.1016/j.ces.2014.01.032

Linek, V., Moucha, T., \& Sinkule, J. (1996). Gas-liquid mass transfer in vessels stirred with multiple impellers-I. Gas-liquid mass transfer characteristics in individual stages. Chemical Engineering Science, 51 
(12), 3203-3212. doi:10.1016/0009-2509(95)00395-9

Lu, W. M., \& Ju, S. J. (1989). Cavity Configuration, Flooding and Pumping Capacity of Disc-Type Turbines in Aerated Stirred Tanks. Chemical Engineering Science, 44 (2), 333-342. doi:10.1016/0009-2509(89)85070-5

Luo, H., \& Svendsen, H. F. (1996). Theoretical model for drop and bubble breakup in turbulent dispersions. AICHE Journal, 42 (5), 1225-1233. doi:10.1002/aic.690420505

Middleton, J. C., \& Smith, J. M. (2004). Gas-Liquid Mixing in Turbulent Systems Handbook of Industrial Mixing (pp. 585-638): John Wiley \& Sons, Inc.

Nienow, A. W., \& Lilly, M. D. (1979). Power Drawn by Multiple Impellers in Sparged Agitated Vessels. Biotechnology and Bioengineering, 21 (12), 2341-2345. doi:10.1002/bit.260211214

Nienow, A. W., Warmoeskerken, M. M. C. G., Smith, J. M., \& Konno, M. (1985). Flooding/loading transition and the complete dispersal condition in aerated vessels agitated by a Rushton-turbine. Paper presented at the 5th European Conference on Mixing, Würzburg.

Nienow, A. W., Wisdom, D. J., \& Middleton, J. C. (1977). The effect of scale and geometry on flooding, recirculation, and power in gassed stirred vessels . Paper presented at the Second European Conf. on Mixing, Cambridge, England.

Nocentini, M., Fajner, D., Pasquali, G., \& Magelli, F. (1993). Gas-Liquid Mass-Transfer and Holdup in Vessels Stirred with Multiple Rushton Turbines - Water and Water Glycerol Solutions. Industrial $\&$ Engineering Chemistry Research, 32 (1), 19-26. doi:10.1021/ie00013a003

Nocentini, M., Magelli, F., Pasquali, G., \& Fajner, D. (1988). A Fluid-Dynamic Study of a Gas-Liquid, Non-Standard Vessel Stirred by Multiple Impellers. Chemical Engineering Journal and the Biochemical Engineering Journal, 37 (1), 53-59. doi:10.1016/0300-9467(88)80006-6

Prince, M. J., \& Blanch, H. W. (1990). Transition Electrolyte Concentrations for Bubble Coalescence. AICHE Journal, 36 (9), 1425-1429. doi:10.1002/aic.690360915

Scargiali, F., D'Orazio, A., Grisafi, F., \& Brucato, A. (2007). Modelling and simulation of gas-liquid hydrodynamics in mechanically stirred tanks. Chemical Engineering Research \& Design, 85 (A5), 637-646. doi:10.1205/cherd06243

Smith, J. M., Warmoeskerken, M. M. C. G., \& Zeef, E. (1987). Flow conditions in vessels dispersing gases in liquids with multiple impellers. Biotechnology Processes , 107-115.

Wang, H. N., Jia, X. Q., Wang, X., Zhou, Z. X., Wen, J. P., \& Zhang, J. L. (2014). CFD modeling of hydrodynamic characteristics of a gas-liquid two-phase stirred tank. Applied Mathematical Modelling, 38 (1), 63-92. doi:10.1016/j.apm.2013.05.032

Warmoeskerken, M. M. C. G., \& Smith, J. M. (1985). Flooding of Disk Turbines in Gas-Liquid Dispersions a New Description of the Phenomenon. Chemical Engineering Science, 40 (11), 2063-2071. doi:10.1016/00092509(85)87023-8

Warmoeskerken, M. M. C. G., \& Smith, J. M. (1988). Impeller loading in multi-turbine vessels. Paper presented at the Proceedings of International Conference on Bioreactor Fluid Dynamics.

Witz, C., Treffer, D., Hardiman, T., \& Khinast, J. (2016). Local gas holdup simulation and validation of industrial-scale aerated bioreactors. Chemical Engineering Science, 152 , 636-648. doi:10.1016/j.ces.2016.06.053

Figure 1

Figure 2

Figure 3 
Figure 4

Figure 5

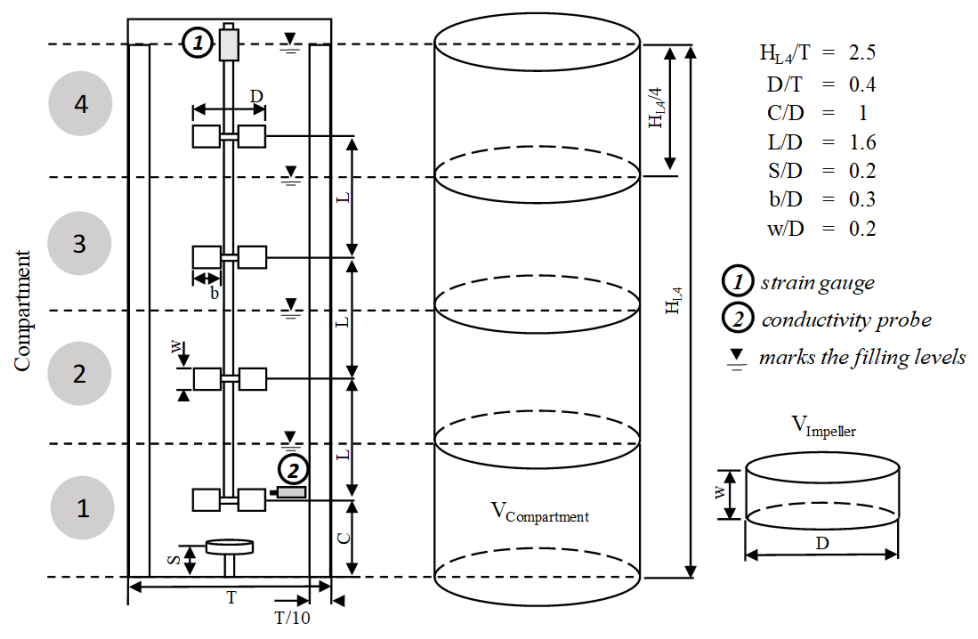



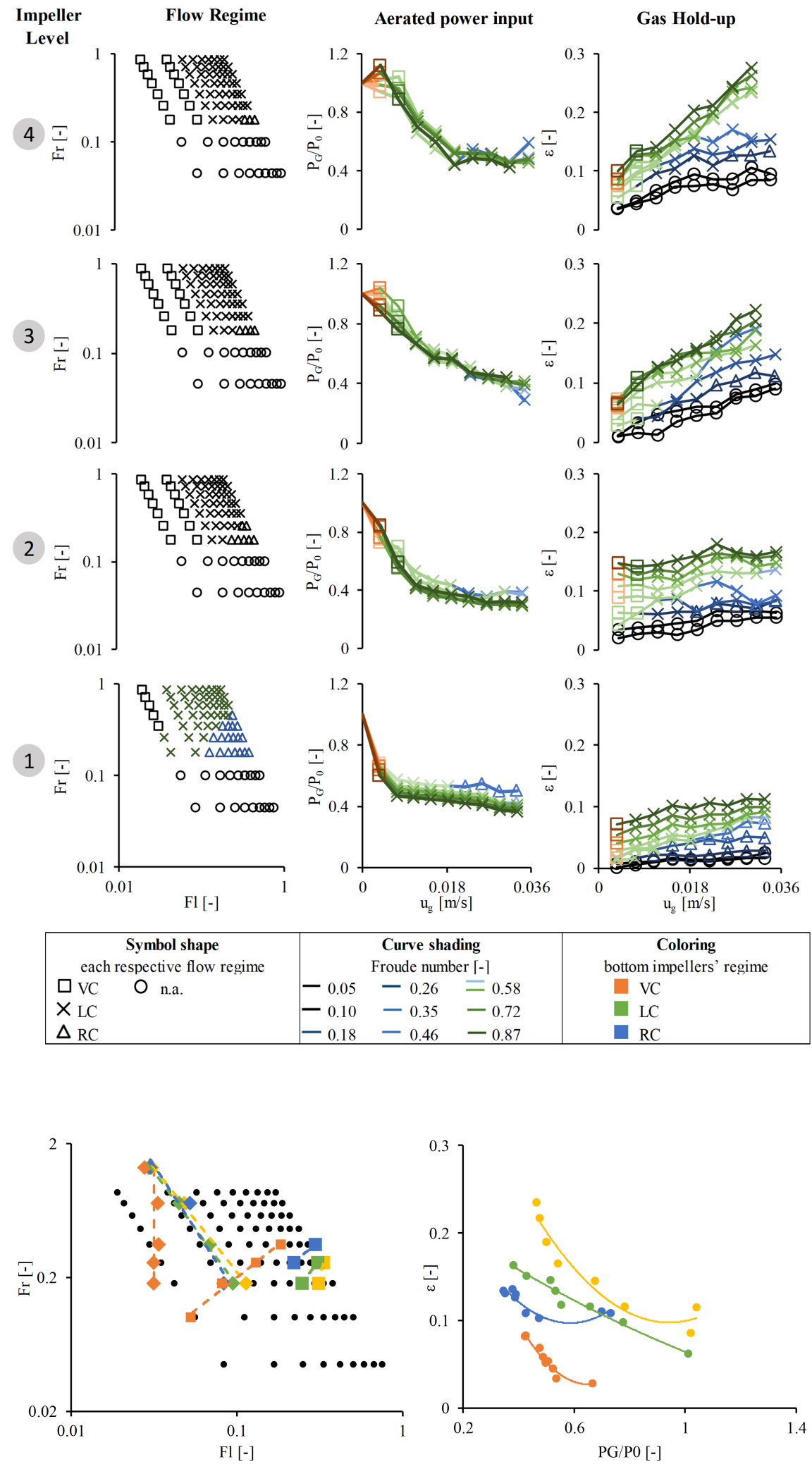

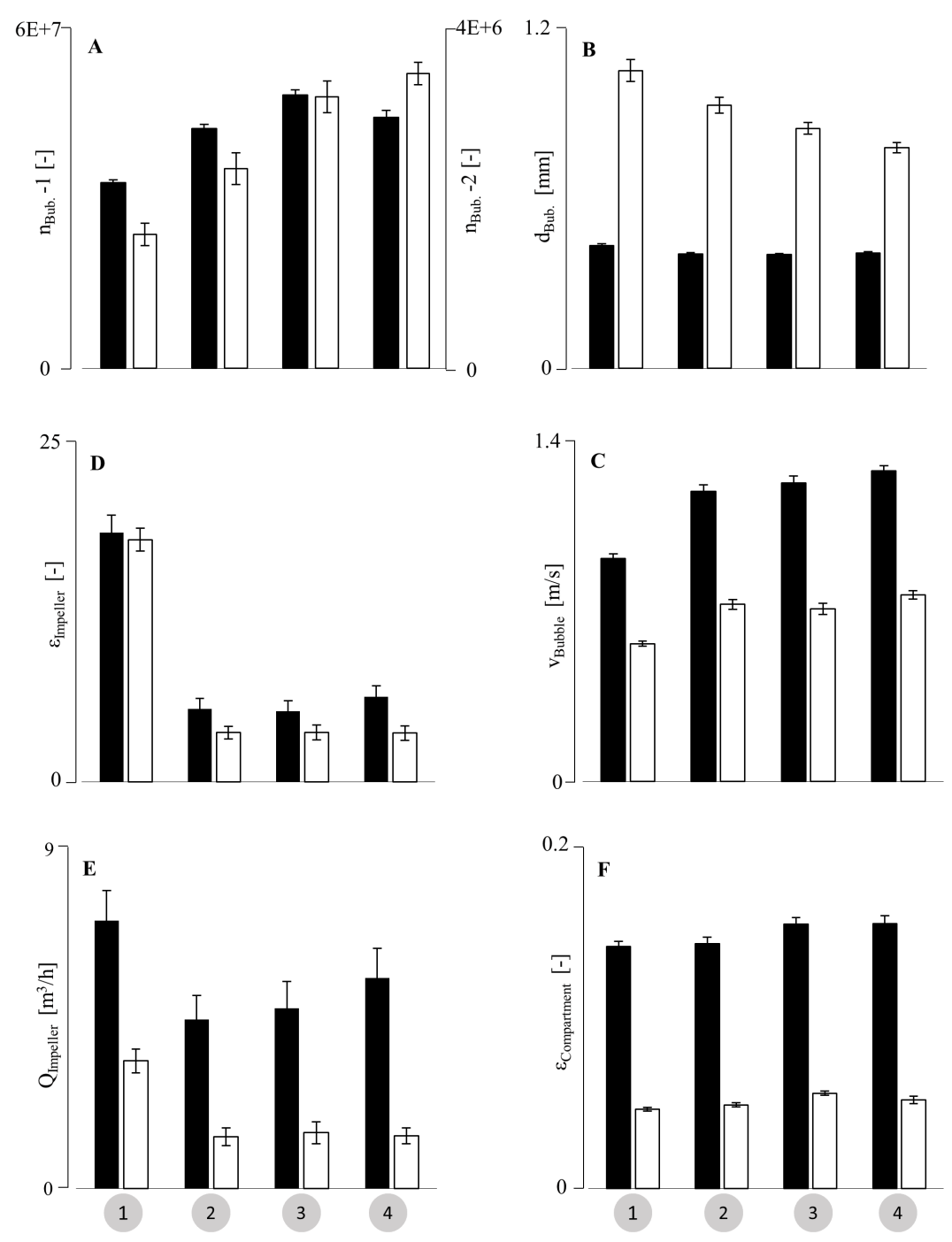


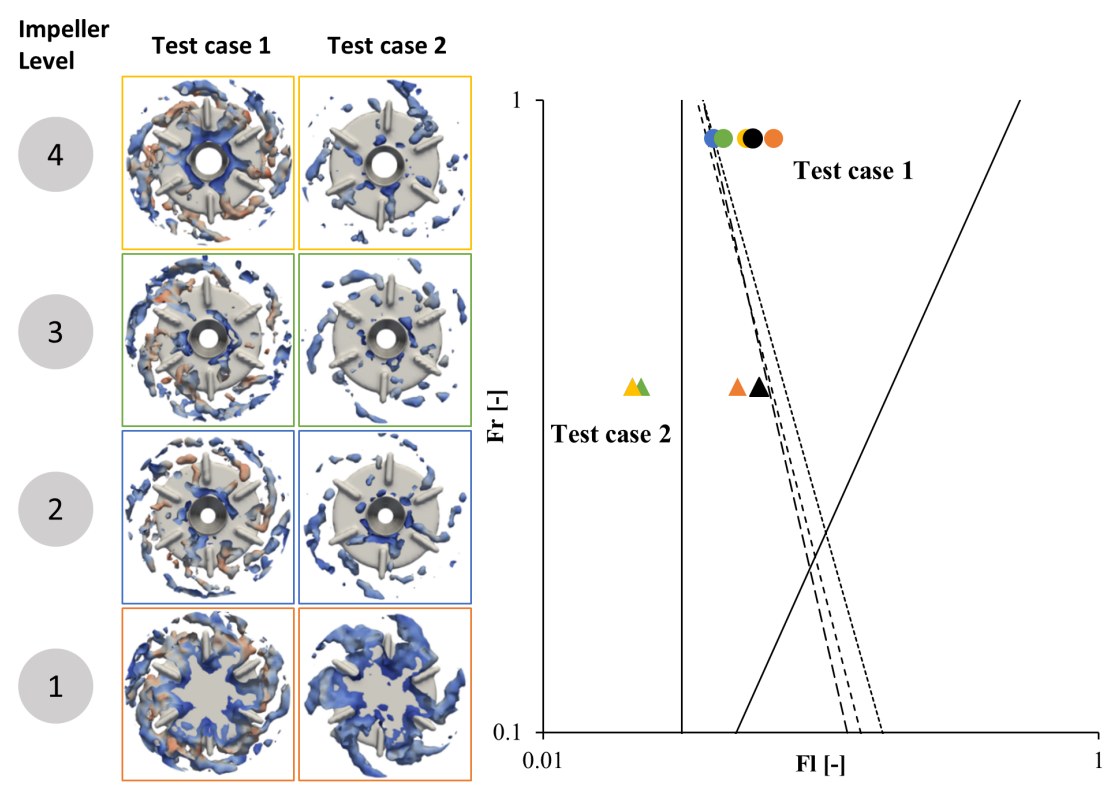

